\title{
Central limit theorems for correlated variables: some critical remarks
}

\author{
H. J. Hilhorst* \\ Laboratoire de Physique Théorique, Bâtiment 210 \\ Univ Paris-Sud XI and CNRS, 91405 Orsay, France ${ }^{\dagger}$
}

(Received on 9 January, 2009)

\begin{abstract}
In this talk I first review at an elementary level a selection of central limit theorems, including some lesser known cases, for sums and maxima of uncorrelated and correlated random variables. I recall why several of them appear in physics. Next, I show that there is room for new versions of central limit theorems applicable to specific classes of problems. Finally, I argue that we have insufficient evidence that, as a consequence of such a theorem, $q$-Gaussians occupy a special place in statistical physics.
\end{abstract}

Keywords: Central limit theorems, Sums and maxima of correlated random variables, $q$-Gaussians

Central limit theorems play an important role in physics, and in particular in statistical physics. The reason is that this discipline deals almost always with a very large number $N$ of variables, so that the limit $N \rightarrow \infty$ required in the mathematical limit theorems comes very close to being realized in physical reality. Before looking at some hard questions, let us make an inventory of a few things we know.

\section{SUMS OF RANDOM VARIABLES}

\section{Gaussians and why they occur in real life}

Let $p(x)$ be an arbitrary probability distribution of zero mean. Draw from it independently $N$ variables $x_{1}, x_{2}, \ldots, x_{N}$ and then ask what is the probability $P_{N}(Y)$ that the scaled sum $\left(x_{1}+x_{2}+\ldots+x_{N}\right) / N^{1 / 2}$ take the value $Y$. The answer, as we explain to our students, is obtained by doing the convolution $P_{N}(Y)=p\left(x_{1}\right) * p\left(x_{2}\right) * \cdots p\left(x_{N}\right)$. After some elementary rewriting one gets

$$
P_{N}(Y)=\frac{1}{\sqrt{2 \pi\left\langle x^{2}\right\rangle}} \exp \left(-\frac{Y^{2}}{2\left\langle x^{2}\right\rangle}+\frac{1}{N^{1 / 2}}[\ldots]\right),
$$

where the dots stand for an infinite series of terms that depend on all moments of $p(x)$ higher than the second one, $\left\langle x^{3}\right\rangle,\left\langle x^{4}\right\rangle$, $\ldots$ In the limit $N \rightarrow \infty$ the miracle occurs: the dependence on these moments disappears from (1) and we find the Gaussian $P_{\infty}(Y)=\left(2 \pi\left\langle x^{2}\right\rangle\right)^{1 / 2} \exp \left(-\frac{1}{2} Y^{2} /\left\langle x^{2}\right\rangle\right)$.

The important point is that even if you didn't know beforehand about its existence, this Gaussian results automatically from any initially given $p(x)$ - for example a binary distribution with equal probability for $x= \pm 1$. This is the Central Limit Theorem (CLT); it says that the Gaussian is an attractor [1] under addition of independent identically distributed random variables. An adapted version of the Central Limit Theorem remains true for sufficiently weakly correlated variables.

This theorem of probability theory is, first of all, a mathematical truth. In order to see why it is relevant to real

\footnotetext{
*Electronic address: Henk.Hilhorsteth.u-psud.fr

$\dagger$ text at the basis of a talk presented at the 7th International Conference on Nonextensive Statistical Mechanics: Foundations and Applications (NEXT2008), Foz do Iguaçu, Paraná, Brazil, 27-31 October 2008.
}

life, we have to examine the equations of physics. It appears that these couple their variables, in most cases, only over short distances and times, so that the variables are effectively independent. This is the principal reason for the ubiquitous occurrence of Gaussians in physics (Brownian motion) and beyond (coin tossing). Inversely, the procedure of fitting a statistical curve by a Gaussian may be considered to have a theoretical basis if the quantity represented can be argued to arise from a large number of independent contributions, even if these cannot be explicitly identified.

\section{Lévy distributions}

Symmetric Lévy distributions. Obviously the calculation leading to (1) requires that the variance $\left\langle x^{2}\right\rangle$ be finite. What if it isn't? That happens, in particular, when for $x \rightarrow \pm \infty$ the distribution behaves as $p(x) \simeq c_{ \pm}|x|^{-1-\alpha}$ for some $\alpha \in(0,2)$.

Well, then there is a different central limit theorem. The attractor is a Lévy distribution $L_{\alpha, \beta}(Y)$, where $Y$ is again the sum of the $x_{n}$ scaled with an appropriate power of $N$ and where $\beta \in(0,1)$ depends on the asymmetry between the amplitudes $c_{+}$and $c_{-}$. A description of the $L_{\alpha, \beta}$ is given, e.g., by Hughes ([2], see $\S 4.2-4.3)$. In the symmetric case $c_{+}=c_{-}$we have $\beta=0$. Then $L_{1,0}(Y)$ is the Lorentz-Cauchy distribution $P_{\infty}(Y)=1 /\left[\pi\left(1+Y^{2}\right)\right]$ and $L_{2,0}(Y)$ is the Gaussian discussed above.

Asymmetric Lévy distributions. If $c_{-}=0$, that is, if $p(x)$ has a slow power law decay only for large positive $x$, then we have $\beta=1$ and the limit distribution is the one-sided Lévy distribution. In the special case $\alpha=\frac{1}{2}$, shown in Fig. 1, we have the Smirnov distribution $L_{\frac{1}{2}, 1}$. It has the explicit analytic expression

$$
P_{\infty}(Y)=\frac{1}{\sqrt{4 \pi Y^{3}}} \exp \left(-\frac{1}{4 Y}\right), \quad Y>0,
$$

and decays for large $Y$ as $\sim Y^{-3 / 2}$.

All these Lévy distributions are attractors under addition of random variables, just like the Gaussian, and each has its own basin of attraction.

\section{Addition of nonidentical variables}

Mathematicians tell us that there do not exist any other attractors, at least not for sums of independent identically distributed (i.i.d.) variables. However, suppose you add inde- 


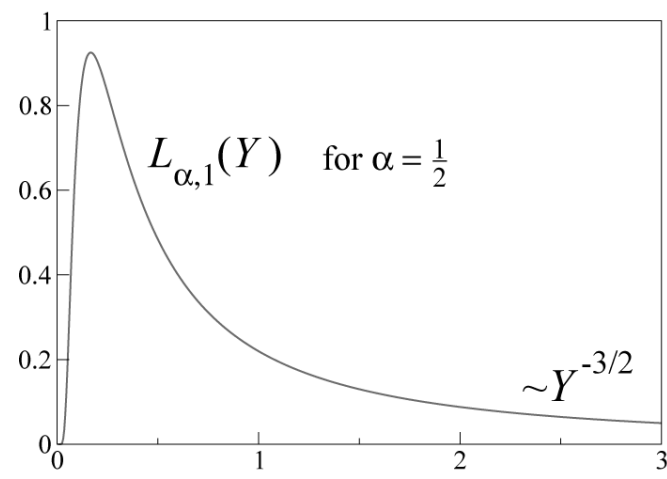

FIG. 1: The one-sided Lévy distribution $L_{\frac{1}{2}, 1}(Y)$ is one possible outcome of a central limit theorem.

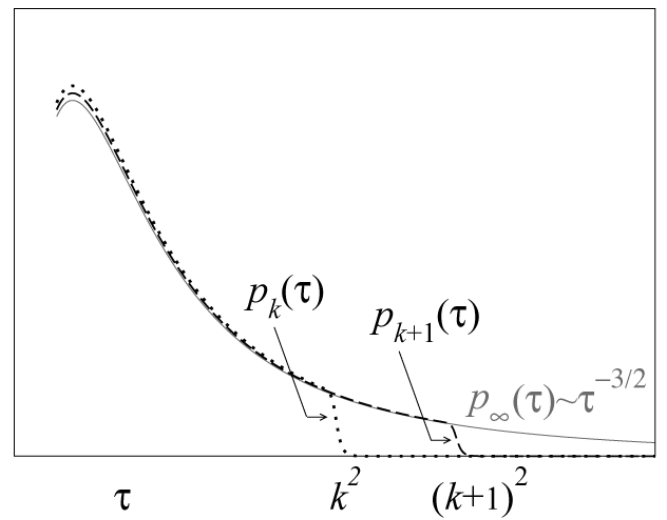

FIG. 2: A sequence of probability distributions developing a "fat tail" proportional to $\sim \tau^{-3 / 2}$ when $k \rightarrow \infty$.

pendent but non-identical variables. If they're not too nonidentical, you still get Gauss and Lévy distributions. The precise premises (the "Lindeberg condition"), under which the sum of a large number of nonidentical variables is Gaussian distributed, may be found in a recent review for physicists by Clusel and Bertin [3].

Now consider a case in which the Lindeberg condition does not hold. Suppose a distribution $p_{\infty}(\tau)$, defined for $\tau>0$, decays as $\tau^{-3 / 2}$ in the large- $\tau$ limit. Let it be approximated, as shown in Fig. 2, by a sequence of truncated distributions $p_{1}(\tau), p_{2}(\tau), \ldots, p_{k}(\tau), \ldots$ which is such that in the limit of large $k$ the distribution $p_{k}(\tau)$ has its cutoff at $\tau \sim k^{2}$. Then how will the sum $t_{L} \equiv \tau_{1}+\tau_{2}+\ldots+\tau_{L}$ be distributed?

The answer is that it will be a bell-shaped distribution which is neither Gaussian nor asymmetric Lévy, but something in between. It's given by a complicated integral that I will not show here. It is again an attractor: it does not depend on the shape of $p(\tau)$ and the $p_{k}(\tau)$ for finite $\tau$, but only on the asymptotic large- $\tau$ behavior of these functions as well as on how the cutoff progresses for asymptotically large $k$.

An example: support of $1 D$ simple random walk. An example of just these distributions occurs in the following not

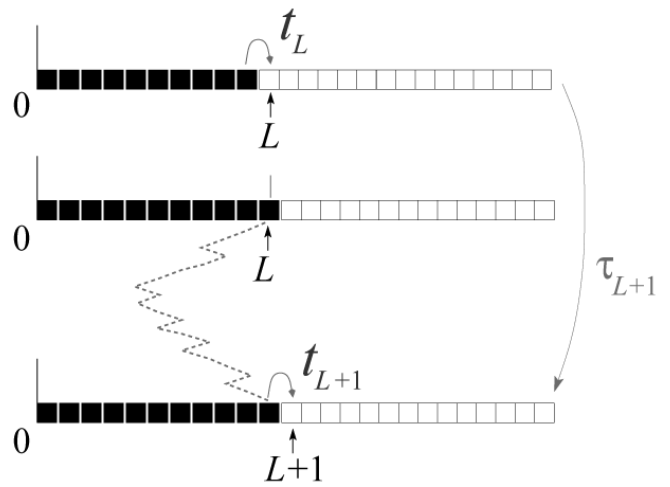

FIG. 3: A random walker on the positive half-line pays its first visit to site $L$ at time $t_{L}$. The sites (squares) already visited are colored black. The time interval between the first visits to $L$ and to $L+$ 1 is equal to $\tau_{L+1}$. During this time interval the walker makes an excursion in the direction of the origin, as indicated by the dotted trajectory. The probability distributions $p_{L}\left(\tau_{L}\right)$ are independent but non-identical.

totally unrealistic situation, depicted in Fig. 3, and which was studied by Hilhorst and Gomes [4]. A random walker on a one-dimensional lattice with reflecting boundary conditions in the origin visits site $L$ for the first time at time $t_{L}$. We can then write $t_{L}=\tau_{1}+\tau_{2}+\ldots+\tau_{L}$, where $\tau_{k}$ is the time difference between the first visit to the $(k-1)$ th site and the $k$ th site. As $k$ increases, the $\tau_{k}$ tend to increase because of longer and longer excursions inside the region already visited. The $\tau_{k}$ are independent variables of the type described above. For $L \rightarrow \infty$ the probability distribution of $t_{L}$ tends to an asymmetric bellshaped function of the scaling variable $t_{L} / L^{2}$ which is neither Gaussian nor Lévy. It is given by an integral that we will not present here. It is universal in the sense that it depends only on the asymptotic large $\tau$ behavior of the functions involved.

\section{Sums having a random number of terms}

The game of summing variables still has other variations. We may, for example, sum $N$ i.i.d. where $N$ itself is a random positive integer. Let $N$ have a distribution $\pi_{N}(v)$ where $v$ is a continuous parameter such that $\langle N\rangle=v$. Then for $v \rightarrow \infty$ one easily derives new variants of the Central Limit Theorem.

\section{MAXIMA OF RANDOM VARIABLES}

\section{Gumbel distributions}

Let us again start from $N$ independent identically distributed variables, but now ask a new question. Let there be a given a probability law $p(x)$ which for large $x$ decays faster than any power law (it might be a Gaussian). And suppose we draw $N$ independent random values $x_{1}, \ldots, x_{N}$ from this law. We will set $Y=\max _{1 \leq i \leq N} x_{i}$. Then what is the probability distribution $P_{N}(Y)$ of $Y$ in the limit $Y \rightarrow \infty$ ? The expression 


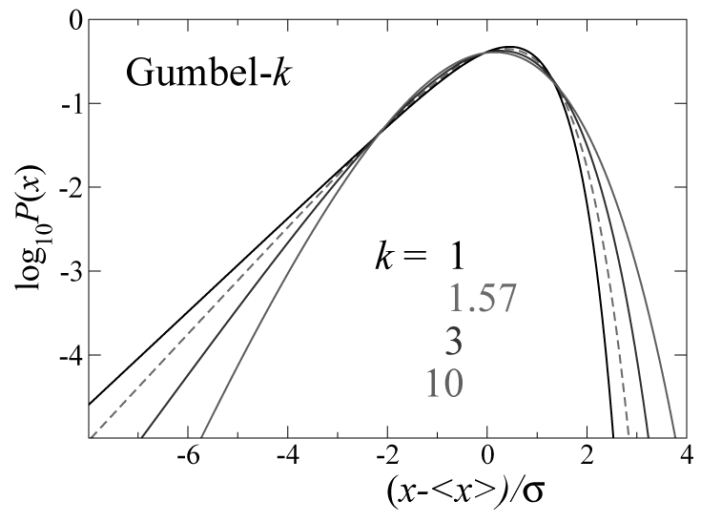

FIG. 4: The Gumbel- $k$ distribution for various values of $k$.

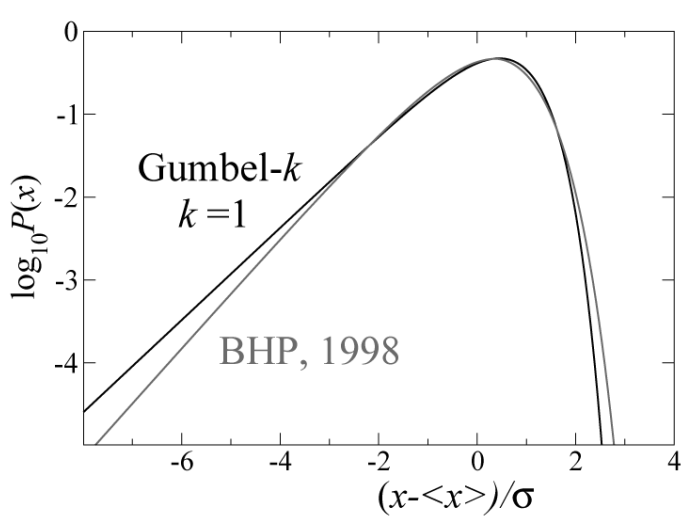

FIG. 5: The Gumbel-1 and the BHP distribution.

is easily written down as an integral,

$$
P_{N}(Y)=\frac{\mathrm{d}}{\mathrm{d} Y}\left(1-\int_{Y}^{\infty} \mathrm{d} x p(x)\right)^{N} .
$$

The calculation is a little harder to do than for the case of a sum. Let us subject the variable $Y$ to an appropriate (and generally $N$-dependent) shift and scaling and again call the result $Y$. Then one obtains

$$
P_{\infty}(Y)=\mathrm{e}^{-Y} \mathrm{e}^{-\mathrm{e}^{-Y}},
$$

which is the Gumbel distribution.

The asymptotic decay of $p(x)$ was supposed here faster than any power law. If it is as a power law, a different distribution appears, called Fréchet; and if $p(x)$ is strictly zero beyond some cutoff $x=x_{\mathrm{c}}$, a third distribution appears, called Weibull. Again, mathematicians tell us that for this new question these three cases exhaust all possibilities.

In Ref. [3] an interesting connection is established between distributions of sums and of maxima.

The Gumbel-k distribution. The Gumbel distribution (4) is depicted in Figs. 4, where it is called "Gumbel-1". This is because we may generalize the question and ask not how the largest one of the $x_{i}$, but how the $k$ th largest one of them is distributed? The answer is that it is a Gumbel distribution of index $k$. Its analytic form is known and contains $k$ as a parameter. For $k \rightarrow \infty$ it tends to a parabola, that is, to a Gaussian.

All these distributions are attractors under the maximum operation. Even if you did not know them in advance, you would be led to them starting from an arbitrary given distribution $p(x)$ within its basin of attraction.

Bertin and Clusel $[5,6]$ show that the definition of the Gumbel- $k$ distribution may be extended to real $k$. These authors also show how Gumbel distributions of arbitrary index $k$ may be obtained as sums of correlated variables. Their review article [3] is particularly interesting.

\section{The BHP distribution}

In 1998 Bramwell, Holdsworth, and Pinton (BHP) [7] adopted a semi-empirical approach to the discovery of new

universal distributions. These authors noticed that, within error bars, exactly the same probability distribution is observed for (i) the experimentally measured power spectrum fluctuations of 3D turbulence; and (ii) the Monte Carlo simulated magnetization of a 2D XY model on an $L \times L$ lattice at temperature $T<T_{\mathrm{c}}$, in spin wave approximation.

For the XY model Bramwell et al. [8,9] were later able to calculate this distribution. It is given by a complicated integral that I will not reproduce here and is called since the "BHP distribution." Fig. 5 shows it together with the Gumbel-1 distribution [10].

Numerical simulations. How universal exactly is the BHP distribution? Bramwell et al. [8] were led to hypothesize that the BHP occurs whenever you look for the maximum of, not independent, but correlated variables. To test this hypothesis these authors generated a random vector $\vec{x}=\left(x_{1}, \ldots, x_{N}\right)$ of $N$ elements distributed independently according to an exponential, and acted on it with a fixed random matrix $M$ such as to obtain $\vec{y}=M \vec{x}$. By varying $\vec{x}$ for a single fixed $M$ they obtained the distribution of $Y=\max _{1 \leq i \leq N} y_{i}$ and concluded that indeed it was BHP.

However, Watkins et al. [11] showed one year later by an analytic calculation that what appears to be a BHP distribution in reality crosses over to a Gumbel-1 law when $N$ is increased. In this case, therefore, the correlation is irrelevant and the attractor distribution is as for independent variables.

Watkins et al. conclude that "even though subsequent results may show that the BHP curve can result from strong correlation, it need not." This example illustrates the danger of trying to attribute an analytic expression to numerically obtained data.

In later work Clusel and Bertin [3] present heuristic arguments tending to explain why distributions closely resembling the BHP distribution occur so often in physics.

\section{Wider occurrence of Gumbel and BHP?}

The Gumbel and BHP distribution have been advanced to fit curves in situations where their occurrence is not $a$ priori expected. Two examples from the literature that appeared this month illustrate this. Palassini [12] performs Monte 


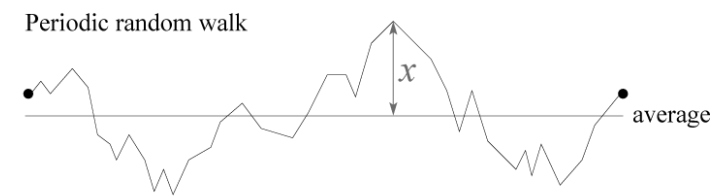

FIG. 6: A random walk on a finite interval with periodic boundary conditions. The random variable $x$ represents the maximum deviation of the walk from its interval average. The probability distribution $P(x)$ was calculated by Majumdar and Comtet [14].

Carlo simulations that yield the ground state energy of the Sherrington-Kirkpatrick model; this author fits his data by a Gumbel-6 distribution ([12], Fig. 4b).

Gonçalves and Pinto [13] consider the distribution of the cp daily return of two stock exchange indices (DJIA30 and S\&P100) over a 21 year period. They find that the cubic root of the square of this distribution is extremely well fitted by the BHP curve ([13], Figs. 1 and 2).

In both cases the authors are right to point out the quality of the fit. But these examples also show that having a very good fit doesn't mean you have a theoretical explanation.

\section{CORRELATED VARIABLES}

In addition to the example discussed above, we will provide here two further examples of how the maximum of a set of correlated random variables may be distributed. These examples will illustrate the diversity of the results that emerge.

Airy distribution. Fig. 6 shows the trajectory of a onedimensional random walker in a given time interval, subject to the condition that the starting point and end point coincide. The walker's positions on two different times are clearly correlated. Let $x$ denote the maximum deviation (in absolute value) of the trajectory from its interval average.

Majumdar and Comtet [14] were able to show that this maximum distance is described by the Airy distribution (distinct from the well-known Airy function), which is a weighted sum of hypergeometric functions that I will not reproduce here. It is again universal: Schehr and Majumdar [15] showed in analytic work, supported by numerical simulations, that this same distribution appears for a wide class of walks with short range steps. It turns out [14], however, that the distribution changes if the periodic boundary condition in time is replaced by free boundaries. This therefore puts a limit on the universality class [1].

Magnetization distribution of Ising $2 D$ at criticality. We consider a finite $L \times L$ two-dimensional Ising model with a set of short-range interaction constants $\left\{J_{k}\right\}$. Its magnetization (per spin) will be denoted $M=N^{-1} \sum_{i=1}^{N} s_{i}$, where $N=L^{2}$ and the $s_{i}$ are the individual spins. We ask what the distribution $P_{L}(M)$ is exactly at the critical temperature $T=T_{\mathrm{c}}$. This distribution can be determined, in principle at least, by a

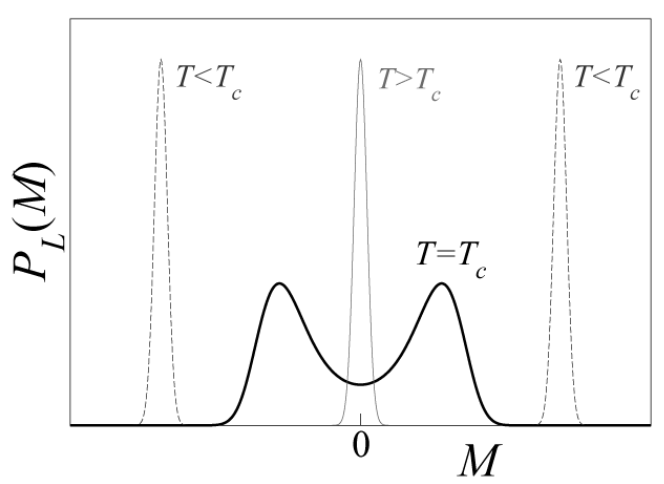

FIG. 7: Qualitative behavior of the distribution $P_{L}(M)$ of the magnetization of the 2D Ising model on a periodic $L \times L$ lattice. The sharp peaks for $T>T_{\mathrm{c}}$ and $T<T_{\mathrm{c}}$ are Gaussians. For $T=T_{\mathrm{c}}$ and under suitable scaling $P_{L}(M)$ tends in the limit $T \rightarrow \infty$ to a double-peaked universal distribution $\mathcal{P}(m)$; see Eq. (5) and the accompanying text.

renormalization calculation which in its final stage gives

$$
L^{-\frac{1}{8}} P_{L}(M)=\mathcal{P}\left(m,\left\{L^{-y_{\ell}} u_{\ell}\right\}\right),
$$

where $m=L^{\frac{1}{8}} M$ and where $\left\{y_{\ell}\right\}$ is a set of positive fixedpoint indices with corresponding scaling fields $\left\{u_{\ell}\right\}$ (i.e. the $u_{\ell}$ are nonlinear combinations of the $J_{k}$ ). In the limit $L \rightarrow$ $\infty$ the dependence on these scaling fields disappears and we have, in obvious notation, that $\lim _{L \rightarrow \infty} L^{-\frac{1}{8}} P_{L}(M)=\mathcal{P}(m)$.

In Fig. 7 the distribution $P_{L}(M)$ is depicted qualitatively for $L \gg 1$ (it has two peaks!), together with the Gaussians that prevail when $T \neq T_{\mathrm{c}}$. The reason for $M$ not being Gaussian distributed exactly at the critical point is that for $T=T_{\mathrm{c}}$ the spin pair correlation does not have an exponential but rather a slow power law decay with distance: the spins are strongly correlated random variables.

The similarity between Eq. (5) and Eq. (1) is not fortuitous: the coarse-graining of the magnetization which is implicit in renormalization, amounts effectively to an addition of spin variables; and the set of irrelevant scaling fields $\left\{u_{\ell}\right\}$ plays the same role as the set of higher moments $\left\{\left\langle x^{n}\right\rangle \mid n \geq 3\right\}$ in Eq. (1).

Eq. (5) says that $\mathcal{P}(m)$ is an attractor under the renormalization group flow; it is reached no matter what set of coupling constants $\left\{J_{k}\right\}$ was given at the outset. Here, too, there are limits on the basin of attraction: the shape of $\mathcal{P}(m)$ depends, in particular, on the boundary conditions (periodic, free, or otherwise [16]).

The conclusion from everything above is that attractor distributions come in all shapes and colors, and that it makes sense to try and discover new ones.

\section{4. $q$-GAUSSIANS}

A $q$-Gaussian $G_{q}(x)$ is the power of a Lorentzian,

$$
G_{q}(x)=\frac{\text { cst }}{\left[1+a x^{2}\right]^{p}}=\frac{\text { cst }}{\left[1+(q-1) x^{2}\right]^{\frac{1}{q-1}}},
$$




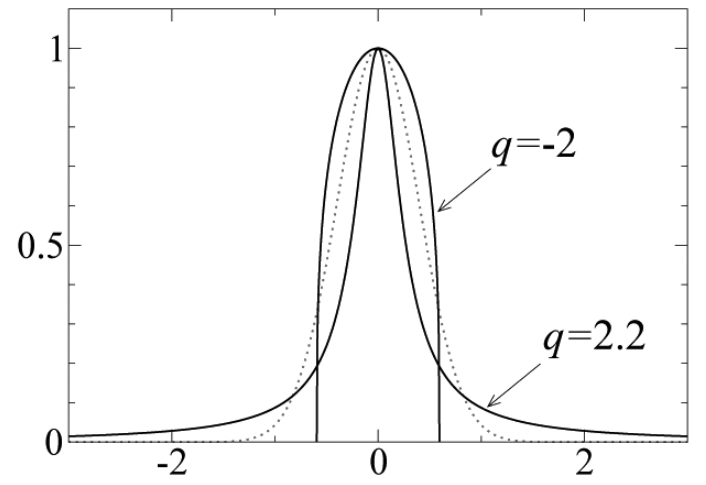

FIG. 8: Examples of $q$-Gaussians. Dotted curve: the ordinary Gaussian, $q=1$. Solid curves: the $q$-Gaussians for $q=2.2$ and $q=-2$; the former has fat tails whereas the latter is confined to a compact support. All three curves are normalized to unity in the origin.

where in the second equality we have set $p=1 /(q-1)$ and scaled $x$ such that $a=q-1$. Examples of $q$-Gaussians are shown in Fig. 8. For $q=2$ the $q$-Gaussian is a Lorentzian; in the limit $q \rightarrow 1$ it reduces to the ordinary Gaussian; for $q<1$ it is a function with compact support, defined only for $-x_{\mathrm{m}}<x<x_{\mathrm{m}}$ where $x_{\mathrm{m}}=1 / \sqrt{1-q}$. For $q=0$ it is an arc of a parabola and for $q \rightarrow-\infty$ (with suitable rescaling of $x$ ) it tends to a rectangular block.

Interest in $q$-Gaussians in connection with central limit theorems stems from the fact that they have many remarkable properties that generalize those of ordinary Gaussians. One may consider, for example, the multivariate $q$-Gaussian obtained by replacing $x^{2}$ in (6) with $\sum_{\mu, v=1}^{n} x_{\mu} A_{\mu v} x_{v}$ (with $A$ a symmetric positive definite matrix). Upon integrating this $q$-Gaussian on $m$ of its variables we find that the marginal $(n-m)$-variable distribution is $q_{m}$-Gaussian with $q_{m}=1-$ $2(1-q) /[2+m(1-q)]$ (see Vignat and Plastino [17]; this relation seems to have first appeared in Mendes and Tsallis [18]).

A special case is the uniform probability distribution inside an $n$-dimensional sphere of radius $R$,

$$
P_{n}\left(x_{1}, \ldots, x_{n}\right)=\operatorname{cst} \times \theta\left(R^{2}-\sum_{\mu=1}^{n} x_{\mu}^{2}\right),
$$

where $\theta$ denotes the Heaviside step function. This is actually a multivariate $q$-Gaussian with $q=-\infty$. Integrating on $m$ of its variables yields a $q$-Gaussian with $q_{m}=1-2 / m$. We see that for large $m$ both in the general and in the special case $q_{m}$ approaches unity and hence these marginal distributions tend under iterated tracing to an ordinary Gaussian shape.

Let us first see, now, how $q$-Gaussians may arise as solutions of certain partial differential equations in physics.

\section{Differential equations and $q$-Gaussians}

Thermal diffusion in a potential. The standard FokkerPlanck (FP) equation describing a particle of coordinate $x$ dif- fusing at a temperature $T$ in a potential $U(x)$ reads

$$
\frac{\partial P(x, t)}{\partial t}=\frac{\partial}{\partial x}\left[U^{\prime}(x) P+k_{B} T \frac{\partial P}{\partial x}\right] .
$$

Its stationary distribution $P_{U}^{\text {st }}(x)$ is the Boltzmann equilibrium in that potential, $P_{U}^{\mathrm{st}}(x)=\mathrm{cst} \times \exp [-\beta U(x)]$, where $\beta=$ $1 / k_{B} T$. For the special choice of potential $U^{\prime}(x)=\alpha x /(1+$ $\left.\gamma x^{2}\right)$ the stationary distribution becomes the $q$-Gaussian

$$
P_{U}^{\mathrm{st}}(x)=\operatorname{cst} \times\left(1+\gamma x^{2}\right)^{-\alpha \beta / \gamma} .
$$

This distribution is an attractor under time evolution, the latter being defined by the FP equation (8); a large class of reasonable initial distributions will tend to (9) as $t \rightarrow \infty$ [19]. It should be noted, however, that by adjusting $U(x)$ we may obtain any desired stationary distribution, and hence the $q$ Gaussian of Eq. (9) plays no exceptional role.

The following observation is trivial but will be of interest later on in this talk. Let $x(t)$ be the Brownian trajectory of the diffusing particle. Let $x(0)$ be arbitrary and let $x(t)$, for $t>0$, be the stochastic solution of the Langevin equation associated [20] with the FP equation (8). Let $\xi_{n}=x(n \tau)-x((n-1) \tau)$, where $\tau$ is a finite time interval. Then $Y_{N}=\xi_{1}+\xi_{2}+\ldots+\xi_{N}$ (without any scaling) is a sum which for $N \rightarrow \infty$ has the distribution $P_{U}^{\text {st }}(Y)$. In particular, if $U(x)$ is chosen such as to yield (9), we have constructed a $q$-Gaussian distributed sum.

Finite difference scheme [21]. Rodríguez et al. [22] recently studied the linear finite difference scheme

$$
r_{N, n}+r_{N, n+1}=r_{N-1, n}
$$

where $N=0,1,2, \ldots$ and $n=0,1, \ldots, N$. The quantity $p_{N, n} \equiv$ $\left(\begin{array}{l}N \\ n\end{array}\right) r_{N, n}$ may be interpreted as the probability that a sum of $N$ identical correlated binary variables be equal to $n$. For specific boundary conditions, the authors were quite remarkably able to find a class of analytic solutions to Eq. (10) and observed that the $N \rightarrow \infty$ limit of the sum law $p_{N, n}$ is a $q$ Gaussian.

To understand better what is happening here, let us set $t=1 / N, x=1-2 n / N$, and $P(x, t)=N\left(\begin{array}{l}N \\ n\end{array}\right) r_{N, n}$. When expanding Eq. (10) in powers of $N^{-1}$ one discovers [23] that $P(x, t)$ satisfies the Fokker-Planck equation

$$
\frac{\partial P(x, t)}{\partial t}=\frac{1}{2} \frac{\partial^{2}}{\partial x^{2}}\left[\left(1-x^{2}\right) P\right]
$$

for $t>0$ and $-1<x<1$. The "time" $t$ runs in the direction of decreasing $N$. Hence Rodríguez et al. have solved a parabolic equation backward in time and determined, starting from the small- $N$ behavior, what is actually an initial condition at $N=\infty$. It is obvious that $q$-Gaussians are not singled out here: there exists a solution to Eq. (11) for any other initial condition at $t=0$, and concomitantly to Eq. (10) for any desired limit function $p_{\infty, n}$ at $N=\infty$.

Therefore, in this and the preceding paragraph, the occurrence of $q$-Gaussians in connection with Fokker-Planck equations cannot be construed as an indication of a new central limit theorem. 
The porous medium equation. Let us consider a fluid flowing through a porous medium. Three equations of physics provide the basic input for the description of this flow, namely (i) the continuity equation for the fluid density $\rho(\vec{x}, t)$; (ii) Darcy's law, which relates the fluid velocity $\vec{v}$ to its pressure $p$ by $\vec{v}=-\operatorname{cst} \times \vec{\nabla} p$; and (iii) the adiabatic equation of state of the ideal gas. Upon combining these one finds the porous medium equation

$$
\frac{\partial \rho}{\partial t}=\Delta \rho^{2-q}, \quad q=1-C_{p} / C_{v},
$$

where $C_{p} / C_{v}$ is the specific heat ratio. For $q=1$ this equation reduces to the ordinary diffusion equation.

Equation (12) is nonlinear and its general solution, i.e., for an arbitrary initial condition $u(\vec{x}, t)$, cannot be found. It is however possible to find special classes of solutions. One special solution is obtained by looking for solutions that are (i) radially symmetric, i.e., dependent only on $x \equiv|\vec{x}|$; and (ii) scale as $u(\vec{x}, t)=t^{-d b} F\left(x t^{-b}\right)$. After scaling of $x$ and $t$ we obtain the similarity solution

$$
\rho(\vec{x}, t)=\frac{c_{0}}{t^{d b}}\left[1+(q-1) \frac{x^{2}}{t^{2 b}}\right]^{-\frac{1}{q-1}},
$$

in which $b=1 /[d(1-q)+2]$ and where also $c_{0}$ is uniquely defined in terms of the parameters of the equation. Mathematicians (see e.g. [24]) have shown that initial distributions with compact support tend asymptotically towards this similarity solution. The asymptotic behavior (13) is conceivably robust, within a certain range, against various perturbations of the porous medium equation. It is not clear to me if and how this property can be connected to a central limit theorem.

\section{$q$-statistical mechanics}

Considerations from a $q$-generalized statistical mechanics [25-27] have led Tsallis [28] to surmise that in the limit $N \rightarrow$ $\infty$ the sum of $N$ correlated random variables becomes, under appropriate conditions, $q$-Gaussian distributed; that is, on this hypothesis $q$-Gaussians are attractors in a similar sense as ordinary Gaussians. Now, variables can be correlated in very many ways. To fully describe $N$ correlated random variables you need the $N$ variable distribution $P_{N}\left(x_{1}, \ldots, x_{N}\right)$. Taking the limit $N \rightarrow \infty$ requires knowing the set of functions

$$
P_{N}\left(x_{1}, \ldots, x_{N}\right), \quad N=1,2,3, \ldots
$$

In physical systems the $P_{N}$ are determined by the laws of nature; the relative spatial and/or temporal coordinates of the variables, usually play an essential role. The examples of the Ising model and of the Airy distribution show how widely the probability distributions of strongly correlated variables may vary. Hence, in the absence of any elements of knowledge about the physical system that they describe, statements of uniform validity about correlated variables cannot be expected to be very specific.

\section{$q$-Central Limit Theorem}

We now turn to a $q$-generalized central limit theorem ( $q$ CLT) formulated by Umarov et al. [29]. It says, essentially, the following. Given an infinite set of random variables $x_{1}, x_{2}, \ldots, x_{n}, \ldots$, let the first $N$ of them be correlated according to a certain condition $\mathcal{C}_{N}(q)$, where $N=1,2,3, \ldots$. Then the partial sum $Y_{N}=\sum_{n=1}^{N} x_{n}$, after appropriate scaling and in the limit $N \rightarrow \infty$, is distributed according to a $q$-Gaussian. The theorem is restricted to $1<q<2$. The conditions $C_{N}(q)$ are concisely referred to as " $q$-independence" in Ref. [29] and for $q=1$ reduce to the usual condition of random variables being independent. Closer inspection of the theorem prompts two questions.

First, the conditions $\mathcal{C}_{N}(q)$ are difficult to handle analytically. If a theoretical model is defined by means of its $P_{N}\left(x_{1}, \ldots, x_{N}\right)$ for $N=1,2,3, \ldots$, then one would have to check that these satisfy the $\mathcal{C}_{N}(q)$. I am not aware of cases for which this has been possible. In the absence of examples it is hard to see why nature would generate exactly this type of correlations among its variables.

Secondly, the proof of the theorem makes use of " $q$-Fourier space," the $q$-Fourier transform $(q$-FT) having been defined in Ref. [29] as a generalization of the ordinary FT. The $q$-FT has the feature that when applied to a $q$-Gaussian it yields a $q^{\prime}$ Gaussian with $q^{\prime}=(1+q) /(3-q)$, for $1 \leq q<3$. Now the $q$-FT is a nonlinear mapping which appears not to have an inverse [30]. It is therefore unclear at present how the statements of the theorem derived in $q$-Fourier space can be translated back in a unique way to "real" space.

\section{THE SEARCH FOR $q$-GAUSSIANS}

\section{Mean-field models}

Independently of this $q$-CLT Thistleton et al. [31] (see also Ref. [32]) attempted to see a $q$-Gaussian arise in a numerical experiment. These authors defined a system of $N$ variables $x_{i}$, $i=1,2, \ldots, N$, equivalent under permutation. Each variable is drawn from a uniform distribution on the interval $\left(-\frac{1}{2}, \frac{1}{2}\right)$ but the $x_{i}$ are correlated in such a way that $\left\langle x_{j} x_{k}\right\rangle=\rho\left\langle x_{1}^{2}\right\rangle$ for all $j \neq k$, where $\rho$ is a parameter in $(0,1)$ [33]. They considered the sum $Y_{N}=\left(x_{1}+\ldots+x_{N}\right) / N$ and determined its distribution $P(Y)$ in the limit $N \gg 1$. For $\rho=\frac{7}{10}$ the numerical results for $P(Y)$ can be fitted very well by a $q$-Gaussian $G_{q}(Y)$ with $q=-\frac{5}{9}$, shown as the dotted curve in Fig. 9. This system of correlated variables is sufficiently simple that Hilhorst and Schehr [34] were able to do the analytic calculation of the distribution. They found that $Y$ is distributed according to

$$
P(Y)=\left(\frac{2-\rho}{\rho}\right)^{\frac{1}{2}} \exp \left(-\frac{2(1-\rho)}{\rho}\left[\operatorname{erf}^{-1}(2 Y)\right]^{2}\right),
$$

for $-\frac{1}{2}<Y<\frac{1}{2}$ shown as the solid curve in Fig. 9. The difference between the exact curve and the $q$-Gaussian approximation is of the order of the thickness of the lines. More importantly, the calculation of Ref. [34] shows that the distribution of the sum $Y$ varies with the initially given one of the $x_{i}$. This initial distribution may be fine-tuned such as to lead for $N \rightarrow \infty$ to almost any limit function $P(Y)-$ in particular, to a $q$-Gaussian. The existence of $q$-Gaussian distributed sums was already pointed out below Eq. (9) and is no surprise. However, there is, here no more than in the case of the FP equation, any indication that distinguishes $q$-Gaussians from other functions. 


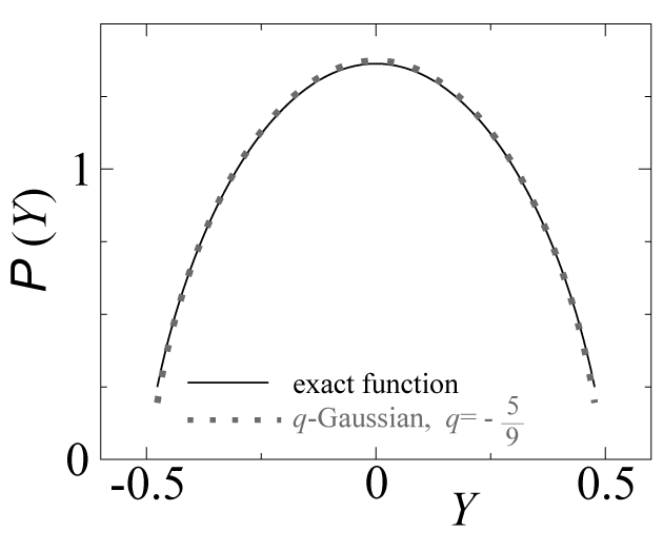

FIG. 9: Comparison of the $q$-Gaussian $G_{q}(Y)$ (dotted curve) guessed in Ref. [31] on the basis of numerical data and the exact distribution $P(Y)$ (Eq. (15), solid curve) calculated in Ref. [34]. The curves are for $\rho=\frac{7}{10}$ and the $q$-Gaussian has $q=-\frac{5}{9}$. The difference between the two curves is of the order of the thickness of the lines and just barely visible to the eye.

The work discussed here concerns a mean-field type model: there is full permutational symmetry between all variables. This will be different in the last two models that we will now take a look at.

\section{Logistic map and HMF model}

Two well-known models of statistical physics have been evoked several times by participants $[35,36]$ at this meeting. The common feature is that in each of them the variable studied is obtained as an average along a deterministic trajectory.

Logistic map. In their search for occurrences of $q$ Gaussians in nature, Tirnakli et al. [37] considered the logistic map

$$
x_{\ell}=a-x_{\ell-1}^{2}, \quad \ell=1,2, \ldots
$$

A motivation for this choice is the appearance [38] of $q$ exponentials in the study of this map. Starting from a uniformly random initial condition $x=x_{0}$, Tirnakli et al. determined the probability distribution of the sum

$$
Y=\sum_{\ell=n_{0}}^{n_{0}+N} x_{\ell}
$$

of successive iterates, scaled with an appropriate power of $N$, in the limit $N \gg 1$. Their initial report of $q$-Gaussian behavior at the Feigenbaum critical point (defined by a critical value $a=a_{\mathrm{c}}$ ) was critized by Grassberger [39]. Inspired by a detailed study due to Robledo and Mayano [40], who connect properties at $a=a_{\mathrm{c}}$ to properties observed on approaching this critical point, Tirnakli et al. [41] took a renewed look at the same question and now see indications for a $q$-Gaussian distribution of $Y$ near the critical point.
Hamiltonian Mean Field Model. The Hamiltonian meanfield model (HMF), introduced in 1995 by Antoni and Ruffo [42], describes $L$ unit masses that move on a circle subject to a mean field potential. The Hamiltonian is, explicitly,

$$
\mathcal{H}=\sum_{i} \frac{p_{i}^{2}}{2}+\frac{1}{2 L} \sum_{i, j}\left[1-\cos \left(\theta_{i}-\theta_{j}\right)\right]
$$

where $p_{i}$ and $\theta_{i}$ are the momentum and the polar angle, respectively, of the $i$ th mass. The angles were originally considered to describe the state of classical XY spins, so that $\vec{m}_{i}=\left(\cos \theta_{i}, \sin \theta_{i}\right)$ is the magnetization of the $i$ th spin. The $\mathrm{HMF}$ has a solvable equilibrium state. At a critical value $U=U_{\mathrm{c}}=0.75$ of the total energy per particle a phase transition occurs from a high-temperature state with uniformly distributed particles to a low-temperature one with a spontaneous value of the "magnetization" $\langle|\vec{M}|\rangle$, where $\vec{M}=L^{-1} \sum_{i=1}^{L} \vec{m}_{i}$. When launched with certain nonequilibrium initial conditions, the system, before relaxing to equilibrium, appears to enter a "quasi-stationary state" (QSS) whose lifetime diverges with $N$. It is impossible to discuss here all the good work that has been done, and is still going on, to attempt to explain the properties of this state (see e.g. Chavanis [43-45], Tsallis et al. [46], Antoniazzi et al. [47], Chavanis et al. [48]). One specific type of numerical simulations, performed by different groups of authors, is relevant for this talk. These have been performed at the subcritical energy $U=0.69$ with initially all particles located at the same point $\left(\theta_{i}=0\right.$ for all $\left.i\right)$ and the momenta $p_{i}$ distributed randomly and uniformly in an interval $\left[-p_{\max }, p_{\max }\right]$. The QSS subsequent to these initial conditions has many features (such as non-Gaussian singleparticle velocity distributions) that have been connected to $q$ statistical mechanics. Of fairly recent interest is the sum $Y_{i}$ of the single-particle momentum $p_{i}(t)$ sampled at regularly spaced times $t=\ell \tau$ along its trajectory,

$$
Y_{i}=\sum_{\ell=n_{0}}^{n_{0}+N} p_{i}(\ell \tau)
$$

The distribution of $Y_{i}$ in the limit of large $N$ is again controversial $[49,50]$. For the specific initial conditions cited above it seems to first approach a fat-tailed distribution, interpreted by some as a $q$-Gaussian, before it finally tends to an ordinary Gaussian.

Comments. The analogy between (17) and (19) is obvious. In both cases the sequence of iterates has long-ranged correlations in the "time" variable $\ell$ and fills phase space in a lacunary way. It is therefore not very surprising that $Y$ and $Y_{i}$ should have non-Gaussian distributions. The $q$-Gaussian shape of these distributions, however, remains speculative. The examples of this talk have shown, on the contrary, that in the absence of specific arguments sums of correlated variables may have a wide variety of distributions. It seems unlikely that haphazard trials will hit exactly on the $q$-Gaussian. 


\section{CONCLUSION}

Universal probability laws occur all around in physics and mathematics, and the quest for them is legitimate and interesting. What lessons can we draw from what precedes?

- It is quite conceivable that new universal distributions may be discovered, either by asking new questions about independent variables; or by asking the traditional questions (sums, maxima,...) about correlated variables.

- Variables may be correlated in an infinity of ways. In the end some real-world input is desirable, be it from physics, finance, or elsewhere.

- Nothing can beat a central limit theorem. A good one, however, should give rise to analytic examples and/or simulation models that reproduce the theorem with high numerical precision.

- In the absence of theoretical arguments, assigning analytic expressions to numerically obtained curves is a risky undertaking.

Let me end by a quotation [51]: "Good theory thrives on reasoned dissent, and [our views] may change in the face of new evidence and further thought."

\section{Acknowledgments}

The author thanks the organizers of NEXT2008 for this possibility of presenting his view. He also thanks Constantino Tsallis for discussions and correspondence over an extended period of time.
[1] In certain contexts attractors are also called universal distributions. In this talk the two terms will be used interchangeably. Concomitantly, "basin of attraction" and "universality class" will denote the same thing here.

[2] B.D. Hughes, Random Walks and Random Environments, Vol. 1: Random Walks, Clarendon Press, Oxford (1995).

[3] M. Clusel and E. Bertin, arXiv:0807.1649.

[4] H.J. Hilhorst and Samuel R. Gomes Jr. (1998), unpublished.

[5] E. Bertin, Phys. Rev. Lett. 95 (2005) 170601.

[6] E. Bertin and M. Clusel, J. Phys. A 39 (2006) 7607.

[7] S.T. Bramwell, P.C.W. Holdsworth, and J.-F. Pinton, Nature 396 (1998) 552

[8] S.T. Bramwell, K. Christensen, J.-Y. Fortin, P.C.W. Holdsworth, H.J. Jensen, S. Lise, J.M. López, M. Nicodemi, J.-F. Pinton, and M. Sellitto, Phys. Rev. Lett. 84 (2000) 3744

[9] S.T. Bramwell, J.-Y. Fortin, P.C.W. Holdsworth, S. Peysson, J.F. Pinton, B. Portelli, and M. Sellitto, Phys. Rev. E 63 (2001) 041106.

[10] The BHP curve is in fact very close to the Gumbel- $k$ distribution with $k=1.57$ shown in Fig. 4.

[11] N.W. Watkins, S.C. Chapman, and G. Rowlands, Phys. Rev. Lett. 89 (2002) 208901.

[12] M. Palassini, J. Stat. Mech. (2008) 10005.

[13] R. Gonçalves and A.A. Pinto, arXiv:0810.2508.

[14] S. Majumdar and A. Comtet, Phys. Rev. Lett. 92 (2004) 225501.

[15] G. Schehr and S. Majumdar, Phys. Rev. E 73 (2006) 056103.

[16] Indeed, $\mathcal{P}(m)$ has been determined for Ising models on surfaces topologically equivalent to a Möbius strip and a Klein bottle! See K. Kaneda and Y. Okabe, Phys. Rev. Lett. 86 (2001) 2134.

[17] C. Vignat and A. Plastino, Phys. Lett. A 365 (2007) 370.

[18] R.S. Mendes and C. Tsallis, Phys. Lett. A 285 (2001) 273.

[19] For $\gamma \rightarrow 0$ the force $-U^{\prime}(x)$ is linear and we recover from (9) the ordinary Gaussian, that is, the equilibrium distribution in a harmonic potential.

[20] N.G. van Kampen, Stochastic Processes in Physics and Chemistry, North-Holland, Amsterdam (1992).

[21] Paragraph added after the Conference.

[22] A. Rodríguez, V. Schwämmle, and C. Tsallis, J. Stat. Mech. (2008) P09006.

[23] H.J. Hilhorst (2009), unpublished.

[24] J.L. Vázquez, The Porous Medium Equation: Mathematical Theory, Oxford University Press, Oxford (2006).

[25] C. Tsallis, J. Stat. Phys. 52 (1988) 479.

[26] M. Gell-Mann and C. Tsallis eds., Nonextensive Entropy - In- terdisciplinary Applications, Oxford University Press, Oxford (2004).

[27] C. Tsallis, Introduction to Nonextensive Statistical Mechanics: Approaching a Complex World, Springer, Berlin (2009).

[28] C. Tsallis, Milan J. Math. 73 (2005) 145.

[29] S. Umarov, C. Tsallis, and S. Steinberg, Milan J. Math. 76 (2008) xxxx.

[30] Ref. [29] associates with a function $f(x)$ the $q$-Fourier transform $\hat{f}_{q}(\xi)=\int \mathrm{d} x f(x)\left[1-(q-1) \mathrm{i} \xi x f^{q-1}(x)\right]^{-1 /(q-1)}$. As an example let us take $f(x)=(\lambda / x)^{1 /(q-1)}$ in an interval $[a, b]$ (with $a, b, \lambda>0$ ) and $f(x)=0$ zero otherwise. Normalization fixes $\lambda$ as a function of $a$ and $b$. Then it is easily verified that $\hat{f}_{q}(\xi)$ is the same for the entire one-parameter family of intervals defined by $\lambda(a, b)=\lambda_{0}$. Hence the $q$-FT is not invertible on the space of probability distributions. Other examples may be constructed.

[31] W. Thistleton, J.A. Marsh, K. Nelson, and C. Tsallis (2006) unpublished; C. Tsallis, Workshop on the Dynamics of Complex Systems Natal, Brazil, March 2007.

[32] L.G. Moyano, C. Tsallis, and M. Gell-Mann, Europhys. Lett. 73 (2006) 813.

[33] The exact procedure that they followed is described in Ref. [34].

[34] H.J. Hilhorst and G. Schehr, J. Stat. Mech. (2007) P06003.

[35] U. Tirnakli, this conference.

[36] A. Rapisarda, this conference.

[37] U. Tirnakli, C. Beck, and C. Tsallis, Phys. Rev. E 75 (2007) 040106(R).

[38] F. Baldovin and A. Robledo, Europhys. Lett. 60 (2002) 518.

[39] P. Grassberger, arXiv:0809.1406.

[40] A. Robledo, this conference; A. Robledo and L.G. Moyano, Phys. Rev. E 77 (2008) 036213.

[41] U. Tirnakli, C. Tsallis, and C. Beck, arXiv:0802.1138.

[42] M. Antoni and C. Ruffo, Phys. Rev. E 52 (1995) 2361.

[43] P.-H. Chavanis, Physica A 365 (2006) 102.

[44] P.-H. Chavanis, Eur. Phys. J. B 52 (2006) 47.

[45] P.-H. Chavanis, Eur. Phys. J. B 53 (2006) 487.

[46] C. Tsallis, A. Rapisarda, A. Pluchino, and E.P. Borges, Physica A 381 (2007) 143.

[47] A. Antoniazzi, D. Fanelli, J. Barré, P.-H. Chavanis, T. Dauxois, and S. Ruffo, Phys. Rev. E, 75 (2007) 011112.

[48] P.-H. Chavanis, G. De Ninno, D. Fanelli, and S. Ruffo, in Chaos, Complexity and Transport: Theory and Applications, Chandre, Leoncini, Zaslavsky Eds., World Scientific, Singapore (2008), p. 3. 
[49] A. Pluchino, A. Rapisarda, and C. Tsallis, Europhys. Lett. 80 (2007) 26002; arXiv:0801.1914.

[50] A. Figueiredo, T.M. Rocha Filho, and M.A. Amato, Europhys. Lett. 83 (2008) 30011.
[51] From the preface of B.J. Baars, A cognitive theory of consciousness, Cambridge University Press, Cambridge (1988). 